\title{
Clinico-Bacteriological Profile of Neonatal Sepsis in a Tertiary Care Hospital
}

\author{
Dr. Urvashi Rana \\ Second Year Resident, Department of Pediatrics, B J Medical College, Ahmadabad, Gujarat \\ Dr. Charul Purani
}

Assistant Professor, Dept. of Pediatrics

Dr. Priyanshi Patel, Dr. Kirti Gupta

Resident, Dept. of Pediatrics

urvashi.rana@gmail.com

\begin{abstract}
Septicemia in new born remains a significant cause of mortality and morbidity in developing countries. Changing bacterial flora and emergence of resistant strains adds to the problem. Thus, neonatal septicemia requires accurate and timely clinical and laboratory diagnosis and proper management for better outcome.

This study was aimed to find out the rate of neonatal sepsis in intramural newborns at our hospital, to correlate clinical profile with bacterial isolates in culture proven sepsis and determine the antimicrobial sensitivity pattern of microbial flora.

The neonates were classified to have early or late onset septicaemia depending on their age at presentation. Risk factors were assessed, essential investigations sent by collecting samples under aseptic precautions. Empirical antimicrobial therapy was started according to antimicrobial guidelines in the NICU. The duration of treatment and hospital stay was noted in all neonates.

During the study period, a total of 142 newborns with suspected sepsis and 67 neonates with culture proven sepsis (47.18\%) were included in the study. Early and late onset sepsis was found in $54.2 \%$ and $45.7 \%$ of cases respectively. Klebsiella spp. and coagulase-negative Staphylococci (CONS) were the most common culture isolates. Majority of the isolates were resistant to Ampicillin, Cefotaxime and Gentamicin.
\end{abstract}

Keywords: Septicaemia, Risk factors, culture isolates, antimicrobial susceptibility

Abbreviations

EOS - Early Onset Sepsis

LOS - Late Onset Sepsis

DIC - Disseminated Intravascular Coagulation

NEC - Necrotizing Enterocolitis

CONS - Coagulase Negative Staphylococci

\section{INTRODUCTION}

Sepsis is the commonest cause of neonatal mortality; responsible for about $30-50 \%$ of the total neonatal deaths in developing countries.1,2 It is estimated that up to $20 \%$ of neonates develop sepsis and approximately $1 \%$ die of sepsis related causes.1 Sepsis related mortality is largely preventable with prevention of sepsis itself, timely recognition, rational antimicrobial therapy and aggressive supportive care.

\section{MATERIALS AND MethodS}

This observational, prospective, single centre study was carried out from October 1, 2014 to September 30, 2015 at a level III NICU in Civil hospital, Ahmedabad, Gujarat, India. A written informed consent was obtained from the child's parent.

All clinically suspected cases of neonatal sepsis admitted in NICU, born intramurally were included. Risk factors for clinical suspicion which were considered in our study: 
At birth:

- Foul smelling liquor.

- Unclean vaginal examination done before delivery

- Duration of labour exceeding 24 hours.

- Birth asphyxia (Apgar<6 at $1 \mathrm{~min}$ ).

- Birth weight $2.5 \mathrm{~kg}$ or less and /or gestation age $<37$ weeks.

- Duration of rupture of membrane before delivery $>24$ hours.

- Maternal pyrexia.

After birth (in NICU or postnatal ward):

- Lethargy

- Refusal to feed

- Abdominal distension

- Respiratory distress

- Temperature instability

- Jaundice

- Convulsion

- Bleeding

- Autonomic instability

Neonates with major congenital malformations and birth weight $<1 \mathrm{~kg}$ and $>4 \mathrm{~kg}$ were excluded from the study.

\section{Methodology}

All neonates were categorized into early onset or late onset sepsis based on day of presentation. Sample for blood culture was sent. An area of approximately $5 \mathrm{~cm}$ over the venipuncture site was disinfected with $70 \%$ alcohol, rubbing vigorously and allowed to dry. This was followed by application of povidine Iodine in concentric circles over the site and allowed to dry for at least 1 minute. About 3-4 ml of blood was drawn using a sterile syringe, out of which $1 \mathrm{ml}$ of the blood sample was inoculated aseptically into a culture bottle containing 5 to $10 \mathrm{ml}$ of culture media. Our hospital has NABL accredited Microbiology laboratory with availability of BACTEC AND BACT/ALERT blood culture system. After collection of blood it is inoculated into a blood culture bottle containing $10 \mathrm{ml}$ of Brain Heart Infusion broth, thus making a dilution of 1 in 10 to nullify the natural bacteriostatic/bactericidal activity of blood. After inoculation, the blood culture bottles were incubated at 37o $\mathrm{C}$ under aerobic conditions in the incubator for 7 days. The inoculated plates were incubated aerobically in the incubator at $37 \mathrm{o} C$ for 24 hours, and the plates were observed for growth. The growth was identified by colony characteristics, gram's stain and standard biochemical tests described in Mackie and McCartney, Practical Medical Microbiology and Bailey and Scott's Diagnostic Microbiology. Cultures which did not yield any growth following three subcultures were reported negative at the end of 7 days. Antimicrobials sensitivity was performed by modified KirbyBauer's Disk Diffusion Method.

\section{Protocols for Giving Antibiotics}

Among early onset sepsis, perinatal risk factors were noted at and after birth.

We used perinatal infection risk score to categorise the neonates for further action of clinical observation, investigation and institution of antimicrobials therapy.

\begin{tabular}{|l|l|l|}
\hline NO. & Perinatalfactor & RiskScore \\
\hline 1 & Foul smelling liquor & 2 \\
\hline 2 & Unclean vaginal examination done before delivery & 2 \\
\hline 3 & Duration of labour exceeding 24 hours & 2 \\
\hline 4 & One minute Apgar score of 0 -6 & 2 \\
\hline 5 & Duration of rupture of membrane before delivery > 24 hours & 1 \\
\hline 6 & Birth weight 2 kg or less and / or gestation less than 37 wks & 1 \\
\hline & TotalScore & 10 \\
\hline
\end{tabular}




\section{Perinatal Infection Risk Score 3,4(By BhaKoo et Al)}

Risk Group category with suggested intervention:

The empirical antimicrobials therapy was started according to antimicrobial guidelines in the NICU.

\begin{tabular}{|l|l|l|}
\hline Total sepsis score & Risk Group & Intervention Suggested \\
\hline $0-3$ & Low Risk & Withhold antimicrobials \\
\hline $4-5$ & Moderate Risk & $\begin{array}{l}\text { Investigate for presence of infection; give } \\
\text { antimicrobials if circumstantial evidence of infection is } \\
\text { present. }\end{array}$ \\
\hline $6-10$ & High Risk & Start Antimicrobials immediately \\
\hline
\end{tabular}

\section{Statistical Analysis}

Data was entered into Microsoft Excel. Analysis of the data was performed using SPSS 20.0. Proportions were compared using Chi-square test of significance. The number of True positive (TP), False positive (FP), True negative (TN) and False negative (FN) results were determined, Sensitivity, Specificity, Positive predictive value and Negative predictive value of various test were calculated. In the above test $p$ value $<0.05$ was accepted as a cut of for statistically significant values.

\section{RESUlTS AND DisCUSSION}

In the present study an attempt has been made to know the incidence of clinically suspected sepsis and culture positive sepsis in our newborn unit, clinical presentation of neonatal sepsis, and various risk factors associated with neonatal septicaemia, bacteriological profile with their antibiogram, to study the outcome of neonatal sepsis in terms of mortality and duration of hospital stay.

Table1. Comparative Study Showing the Incidence of Suspected Sepsis

\begin{tabular}{|c|c|c|c|c|}
\hline Total Live Birth & $\begin{array}{l}\text { Intramural } \\
\text { Neonates Admitted } \\
\text { in NICU }\end{array}$ & $\begin{array}{l}\text { Neonates with } \\
\text { suspected } \\
\text { Sepsis }\end{array}$ & Incidence per 1000 live birth & NNPD 2002-03² \\
\hline 4524 & 778 & 223 & 28.6 & 30 \\
\hline
\end{tabular}

Incidence of suspected sepsis is $28.6 / 1000$ live birth

Similar observation was seen in the studies done by Neonatal and Perinatal data base who reported incidence of sepsis 30/1000 live birth2.

Table2. Comparative Studies Showing Culture Positivity Rate

\begin{tabular}{|l|l|l|l|}
\hline Study group & Year & Total culture positive & Positivity rate \\
\hline Present study & $2014-15$ & $117 / 223$ & $52.61 \%$ \\
\hline NNPD 2002-03 & 2002 & $1248 / 4360$ & $28.6 \%$ \\
\hline Roy et al16 & 2002 & $346 / 728$ & $47.50 \%$ \\
\hline Tallur et al & & $157 / 242$ & $64.87 \%$ \\
\hline Joshi et al $^{9}$ & 2000 & $1326 / 3427$ & $25 \%$ \\
\hline
\end{tabular}

In the present study, 117 of 223 cases studied were culture positive, giving a positivity rate of $52.61 \%$.

Present study was comparable with the studies conducted by Tallur et al8 and Roy et al167, while study conducted by Joshi et al,9 and NNPD4 showed a very low culture positivity.

The culture positivity depends on time of sampling, extent bacteremia in neonate and prior antibiotic treatment in the neonate.

Table3. Comparative Studies Showing the Symptoms

\begin{tabular}{|l|l|l|l|}
\hline Symptoms & Present study (n=223) & Chudgar HH et al $^{\mathbf{1 1}}$ & Shah CP et al $^{\mathbf{1 0}}$ \\
\hline Refusal to feed & $77.84 \%$ & $84 \%$ & $80 \%$ \\
\hline Lethargy & $68.80 \%$ & $42 \%$ & \\
\hline Fever & $59.76 \%$ & $44 \%$ & $30 \%$ \\
\hline Excessive crying & $47.81 \%$ & $21 \%$ & \\
\hline Convulsion & $44.89 \%$ & & $30 \%$ \\
\hline Abdominal distension & $41.89 \%$ & $18 \%$ & $20 \%$ \\
\hline Vomiting & $39.94 \%$ & $26 \%$ & $21 \%$ \\
\hline Diarrhoea & $37.90 \%$ & $13 \%$ & $10 \%$ \\
\hline Jaundice & $33.81 \%$ & & $40 \%$ \\
\hline
\end{tabular}


Urvashi Rana et al.

Refusal to feed was present in $77.84 \%$ of patient as seen with other studies Chudgar HH et al $(84 \%)$ and Shah CP et al (80\%). It is present in most of the sick babies. Change in the feeding pattern, refusal to feed, inattention and lethargy during feeding are the earliest and most common indicator of sepsis.

Table4. Comparative Studies Showing the Signs

\begin{tabular}{|l|l|l|l|}
\hline Signs & Present study (n=223) & Chudgar HH et al $^{\mathbf{1 1}}$ & Shah CP et al $^{\mathbf{1 0}}$ \\
\hline Increased respiratory rate & $65.88 \%$ & $34 \%$ & $60 \%$ \\
\hline Tachycardia & $60.93 \%$ & & \\
\hline Hypothermia & $35.86 \%$ & $13 \%$ & $36 \%$ \\
\hline Sclerema & $34.98 \%$ & $23 \%$ & $36 \%$ \\
\hline Apnoea & $29.73 \%$ & $21 \%$ & $20 \%$ \\
\hline Petechiae & $26.28 \%$ & $19 \%$ & $6 \%$ \\
\hline Cyanosis & $24.78 \%$ & $15 \%$ & \\
\hline Mottling & $21.86 \%$ & $16 \%$ & \\
\hline Bulging AF & & \\
\hline Pallor & $12.24 \%$ & & \\
\hline Hepatosplenomegaly & $10.78 \%$ & & \\
\hline
\end{tabular}

The early clinical features of sepsis are often subtle and non specific and require a high index of suspicion for early diagnosis supported by laboratory investigations.

Respiratory causes were pneumonia, pneumothorax etc. Central causes were pyogenic meningitis, ICH etc. It could be due to metabolic in origin as in acidosis or mechanical with severe abdominal distension.

Signs like increased respiratory rate, hypothermia, sclerema, apnoea, petechiae comparable to Chudgar $\mathrm{HH}$ et al and Shah $\mathrm{CP}$ et al studies.

Table18. Organisms Isolated with Respect to Classification of Sepsis

\begin{tabular}{|l|l|l|l|}
\hline Bacterial isolates & EOS $(\mathbf{n = 5 1})$ & LOS $(\mathbf{n = 6 6 )}$ & TOTAL $(\mathbf{n = 1 1 7})$ \\
\hline Gram positive isolates & $\mathbf{2 0}(\mathbf{3 9 . 2 1 \%})$ & $\mathbf{2 2}(\mathbf{3 3 . 3 3 \% )}$ & $\mathbf{4 2}(\mathbf{3 5 . 8 9 \%})$ \\
\hline Staphylococcus aureus. & $5(9.80 \%)$ & $8(12.12 \%)$ & $13(11.11 \%)$ \\
\hline Coagulase negative staphylococcus & $15(29.41 \%)$ & $14(21.21 \%)$ & $29(24.78 \%)$ \\
\hline Gram negative isolates & $\mathbf{3 1}(\mathbf{6 0 . 7 8 \%})$ & $\mathbf{4 4}(\mathbf{6 6 . 6 6 \%})$ & $\mathbf{7 5}(\mathbf{6 4 . 1 0 \%})$ \\
\hline Klebsiella pneumonia & $19(37.25 \%)$ & $28(23.93 \%)$ & $47(40.17 \%)$ \\
\hline Pseudomonas aeruginosa & $7(13.72 \%)$ & $10(15.15 \%)$ & $17(14.52 \%)$ \\
\hline Escherichia coli & $2(3.92 \%)$ & $4(6.06 \%)$ & $6(5.12 \%)$ \\
\hline Enterobacter & $2(3.92 \%)$ & $1(1.51 \%)$ & $3(2.56 \%)$ \\
\hline Acinetobacter & $1(1.96 \%)$ & $1(0.51 \%)$ & $2(1.72 \%)$ \\
\hline
\end{tabular}

Out of 117 culture positive cases $42(35.78 \%)$ were gram positive isolates and 75 (64.10\%) were gram negative isolates.

Klebsiella pneumonia $(40.17 \%)$ followed by Coagulase negative staphylococcus $(24.78 \%)$ were commonest isolates.

Gram negative isolates were common culprit for both early onset sepsis and late onset sepsis.

Table22. Outcome of Culture Positive Sepsis

\begin{tabular}{|l|l|l|l|l|}
\hline Type of sepsis & Dis-charge & DAMA & expiry & Total \\
\hline EOS & 28 & 1 & 22 & 51 \\
\hline LOS & 37 & 5 & 24 & 66 \\
\hline Total & $65(55.78 \%)$ & $6(5.2 \%)$ & $46(38.94 \%)$ & 117 \\
\hline
\end{tabular}

Out of 117 neonates $55.78 \%$ were discharged, 5.2\% had taken DAMA and 38.94\% were expired.

\section{DisCUSSION}

Neonatal sepsis can be classified into two major categories depending up on the onset of symptoms: 12

(1) Early onset septicemia.

(2) Late onset septicemia. 


\section{Early Onset Sepsis (EOS)}

- It presents within the first 72 hours of life. In severe cases, the neonate may be symptomatic at birth.

- Infants with EOS usually present with respiratory distress and pneumonia. The source of infection is generally the maternal genital tract. The infant may acquire the microbe by passage through a colonized birth canal during delivery.

- Knowledge about these potential risk factors would help in early diagnosis of sepsis.

Based on the studies from India, the following risk factors seem to be associated with an increased risk of early onset sepsis: 12,13

- Low birth weight $(<2500$ grams) or prematurity.

- Febrile illness in the mother with evidence of bacterial infection within 2 weeks prior to delivery.

- Foul smelling and/or meconium stained liquor.

- Rupture of membranes $>24$ hours. Single unclean or $>3$ sterile vaginal examination(s) during labour.

- Prolonged labour (sum of 1st and 2nd stage of labour $>24 \mathrm{hrs}$ ).

- Prenatal asphyxia (Apgar score <4 at 1 minute).

Presence of foul smelling liquor or three of the above mentioned risk factors warrant initiation of antimicrobial therapy. Infants with two risk factors should be investigated and then treated accordingly.

\section{Late Onset Sepsis (LOS)}

- It usually presents after 72 hours of age. The source of infection in LOS is either nosocomial (hospital-acquired) or community-acquired and neonates usually present with septicemia, pneumonia or meningitis. 14,15

- Vector for such colonization include vascular or urinary catheters, other indwelling lines or contact from caregivers with bacterial colonization.

Factors that might increase the risk of community-acquired LOS include:

- Poor hygiene.

- Poor cord care.

- Bottle-feeding.

- Prelacteal feeds.

Various factors that predispose to an increased risk of nosocomial sepsis include:

- Low birth weight.

- Prematurity.

- Admission in intensive care unit.

- Mechanical ventilation.

- Invasive procedures.

- Administration of parenteral fluids, and use of stock solutions.

For a favourable outcome during the management of neonatal infections early diagnosis is crucial. The presence of one or more perinatal risk factors has been shown to have a direct association with septicaemia. Hence attempts have been made to formulate a scoring system based on these factors to predict the risk of outcome the dilemma regarding institution of antimicrobials to the at risk neonates. 3,4

\section{Clinical Features}

The ability of new born to respond infection is limited and the earliest signs of sepsis are often subtle and non specific, a high index of suspicion is needed for early diagnosis. 
A. Non Specific Features:

- Refusal to suck

- Lethargy

- Poor cry

- Temperature instability

- Poor perfusion, prolonged capillary refill time

- Hypotonia, absent neonatal reflexes

- Bradycardia / tachycardia

- Respiratory distress

- Apnea / gasping respiration

- Abdominal distension

\section{B. Specific Features Related to Various Systems:}

a. Central Nervous System (CNS):

- $\Rightarrow \quad$ Bulging anterior fontanel.

- $\ni \quad$ Vacant stare.

- $\Rightarrow$ High pitched cry.

- $\Rightarrow \quad$ Excessive irritability.

- $\Rightarrow \quad$ Seizures.

- $\Rightarrow$ Neck retraction.

b. Cardio Vascular System (CVS):

- $\Rightarrow$ Hypotension.

- $\Rightarrow$ Poor perfusion.

- $\Rightarrow$ Shock.

- $\Rightarrow$ tachycardia

c. Gastrointestinal:

- $\Rightarrow \quad$ Feed intolerance.

- $\Rightarrow$ Vomiting.

- $\Rightarrow$ Diarrhoea.

- $\Rightarrow$ Abdominal distension.

- $\Rightarrow$ Necrotizing enterocolitis (NEC).

d. Hepatobilliry:

- $\Rightarrow$ Hepatomegaly.

- $\Rightarrow$ Pathological hyperbilirubinemia.

e. Renal:

- $\Rightarrow$ Acute renal failure.

f. Haematological:

- $\Rightarrow \quad$ Bleeding.

- $\Rightarrow$ Petechiae.

- $\Rightarrow$ Purpura.

- $\Rightarrow$ Disseminated intravascular coagulation.(DIC) 
g. Skin changes:

- $\Rightarrow \quad$ Multiple pustules.

- $\Rightarrow$ Cyanosis.

- $\Rightarrow$ Abscess.

- $\Rightarrow$ Sclerema.

- $\Rightarrow$ Mottling.

- $\Rightarrow \quad$ Umbilical redness and discharge.

C. The Following Features are Commonly Encountered in the Neonates Suffering from Sepsis:

- Hypothermia

- Hyperthermia/Fever

- Apnea

- Tachypnea/ Respiratory distress

- Bradycardia/ Tachycardia

- Rash

- Hypotonia/ Absent Neonatal Reflexes

- Central Nervous System (co-existing meningitis or ventriculitis)

- Abdominal distension

- Sclerema

- Necrotizing Enterocolitis (NEC):

- Jaundice

- Disseminated intravascular coagulation (DIC)

- Osteomyelitis and septic arthritis

\section{Conclusion}

It is evident from this study that amongst Gram-negative organisms Klebsiella, Pseudomonas, and amongst gram positive organisms CONS, and S. Aureus are the leading cause of neonatal sepsis . Also the most challenging observation was the resistance pattern of these isolates to multiple antibiotics. Formulation of an antibiotic policy in NICU of every hospital is the need of the hour. Furthermore, health education should be provided to all health care providers to promote rational use and to avoid indiscriminate use of antibiotics.

\section{REFERENCES}

[1] Bang AT, Bang RA, Bactule SB, Reddy HM, Deshmukh MD. Effect of home-based neonatal care and management of sepsis on neonatal mortality: field trial in rural India. Lancet 1999; 354:1955-61.

[2] Report of the National Neonatal Perinatal Database (National Neonatology Forum) 2002-03.

[3] BhakooON.Neonatal bacterial infections at Chandigarh:Adecade of experience .Indian J Pediatr 1980;47(388):419-24.

[4] VedParkashTakkar, OmkarNathBhakoo, Anil Narang. Scoring System for the Prediction of Early Neonatal Infections. Indian Pediatrics 1974 Sept; 11 (9): 597-600.

[5] Gupte S. Neonatal septicemia: Issues and controversies (Guest Lecture), Third Workshop of Asian Oceanian Perinatal Societies, Katamandu, Nepal 1997. Recent advances in Pediatrics: Neonatology.1999; 4(sp.vol):151-6.

[6] Anil Narang ,KanyaMukhopadhyay. Protocols in Neonatology 2000 Revised edition:!13-23.

[7] Roy 1, Jain A, Kumar M, Agarwal SK. Bacteriology of Neonatal Septicaemia in a Tertiary care Hospital of Northern India. Indian Journal of Medical Microbiology 2002Jul;20(3): 156-9. 
[8] Tallur SS, Kasturi AV, Shobha D. Nadgir, Krishna BVS. Clinico-bacteriologicai Study of Neonatal Septicemia in Hubli. Indian J Pediatr 2000; 67 (3): 169-

[9] Joshi SG, Ghole VS, Niphadkar KB. Neonatal Gram-Negative Bacteremia. Indian J Pediatr 2000; 67(1): 27-32.

[10] Takkar VP, Bhakoo ON, Narang A. Scoring system for the prediction of early neonatal infections. Indian Pediatr. 1974; 11:597-600.

[11] Gerdes JS. Diagnosis and management of bacterial infection in neonate.PaeditricClinN Am 2004; 51: 939-959.

[12] Singh M, Narang A, Bhakoo ON. Predictive perinatal score in the diagnosis of neonatal sepsis.J TropPediatr. 1994 Dec; 40(6):365-8.

[13] Takkar VP, Bhakoo ON, Narang A. Scoring system for the prediction of early neonatal infections. Indian Pediatr.

[14] Baltimore RS. Neonatal nosocomial infections.SeminPerinatol 1998; 22:25-32.

[15] Wolach B. Neonatal sepsis: pathogenesis and supportive therapy. Semin Perinatol1997; 21:28-38 8. Gerdes JS, Polin R.Early diagnosis and treatment of neonatal sepsis.Indian J Pediatr 1998; 65:63-78. 